ARTICLE

\title{
Transport in helical Luttinger liquids in the fractional quantum Hall regime
}

Ying Wang ${ }^{1}$, Vadim Ponomarenko ${ }^{1,2}$, Zhong Wan ${ }^{10}{ }^{1,6}$, Kenneth W. West $^{3}$, Kirk W. Baldwin ${ }^{3}$, Loren N. Pfeiffer ${ }^{3}$, Yuli Lyanda-Geller ${ }^{1,4 凶}$ \& Leonid P. Rokhinson (10) ${ }^{1,4,5 凶}$

Domain walls in fractional quantum Hall ferromagnets are gapless helical one-dimensional channels formed at the boundaries of topologically distinct quantum Hall (QH) liquids. Naiively, these helical domain walls (hDWs) constitute two counter-propagating chiral states with opposite spins. Coupled to an s-wave superconductor, helical channels are expected to lead to topological superconductivity with high order non-Abelian excitations ${ }^{1-3}$. Here we investigate transport properties of hDWs in the $\nu=2 / 3$ fractional $\mathrm{QH}$ regime. Experimentally we found that current carried by hDWs is substantially smaller than the prediction of the naïve model. Luttinger liquid theory of the system reveals redistribution of currents between quasiparticle charge, spin and neutral modes, and predicts the reduction of the hDW current. Inclusion of spin-non-conserving tunneling processes reconciles theory with experiment. The theory confirms emergence of spin modes required for the formation of fractional topological superconductivity.

\footnotetext{
${ }^{1}$ Department of Physics and Astronomy, Purdue University, West Lafayette, IN, USA. ${ }^{2}$ loffe Physico-Technical Institute, Saint-Petersburg, Russia.

${ }^{3}$ Department of Electrical Engineering, Princeton University, Princeton, NJ, USA. ${ }^{4}$ Birck Nanotechnology Center, Purdue University, West Lafayette, IN, USA.

${ }^{5}$ Department of Electrical and Computer Engineering, Purdue University, West Lafayette, IN, USA. ${ }^{6}$ Present address: Department of Chemistry and

Biochemistry, University of California, Los Angeles, CA, USA. ${ }^{\circledR}$ email: yuli@purdue.edu; leonid@purdue.edu
} 
G apless chiral edge states, a hallmark of the quantum Hall effect (QHE), are formed at the boundaries of the twodimensional (2D) electron liquid. These states are protected due to their topological properties; their spatial separation suppresses backscattering and insures precise quantization of the Hall conductance over macroscopic distances ${ }^{4}$. Symmetryprotected topological systems can support spatially coexisting counter-propagating states. For example, in 2D topological insulators, time reversal symmetry insures orthogonality of Kramers doublets ${ }^{5-7}$; in graphene, the conservation of angular momentum prevents backscattering in the quantum spin Hall effect regime ${ }^{8}$. Local symmetry protection is not as robust as spatial separation in the QHE and, as a result, helical domain walls (hDWs) have finite scattering and localization lengths. Helical states can be also engineered by arranging proximity of two counter-propagating chiral states with opposite polarization, e.g., in electron-hole bilayers ${ }^{9}$ or double quantum well structures ${ }^{10}$, where local charge redistribution between two quantum wells creates two counter-propagating chiral states at the boundary of quantum Hall liquids with different filling factors. In the latter system, spatial separation into two quantum wells suppresses the interchannel scattering, and transport in each chiral channel is found to be ballistic over macroscopic distances.

An intriguing possibility to form helical channels in the interior of a $2 \mathrm{D}$ electron gas is to induce a local quantum Hall ferromagnetic transition. In the integer $\mathrm{QHE}$ regime, scattering between overlapping chiral edges from different Landau levels is suppressed due to the orthogonality of the wavefunctions, but spin-orbit interaction mixes states with opposite spins and opens a small gap in the helical spectrum ${ }^{11-13}$. In the fractional quantum Hall effect (FQHE) regime, an electrostatically-controlled transition between unpolarized $(u)$ and polarized $(p) v=2 / 3$ states results in the formation of a conducting channel at the boundary between $u$ and $p$ regions (filling factor $v^{-1}=B / n \phi_{0}$, where $B$ is an external magnetic field, $\phi_{0}=h / e$ is a flux quanta and $n$ is electron density). Superficially, a transition between $u$ and $p$ states in the bulk can be understood as a crossing of two composite fermion energy states with opposite spins polarization ${ }^{10,14,15}$. Within this model, the hDW at the $u-p$ boundary consists of two counterpropagating chiral states with opposite spin and fractionalized charge excitations, and presents an ideal platform to build fractional topological superconductors with parafermionic and Fibonacci excitations $s^{1,2,16-19}$. Highly correlated $v=2 / 3$ state exhibits rich physics beyond an oversimplified model of $v^{*}=2$ integer QHE for weakly interacting composite fermions and includes observation of upstream neutral modes ${ }^{20-23}$, short-range upstream charge $\operatorname{modes}^{24}$, and a crossover from $e^{*}=1 / 3$ to $e^{*}=2 / 3$ charge excitations in shot noise measurements ${ }^{25}$. In the bulk, the spin transition at $v=2 / 3$ is accompanied by nuclear polarization ${ }^{26-28}$ indicating spin-flip processes in the $2 \mathrm{D}$ gas, a phenomena not seen in bilayer systems ${ }^{10,29}$. Thus, we expect a $\mathrm{hDW}$ formed at a boundary between $u$ and $p v=2 / 3$ states to be more complex than a simple overlap of two noninteracting chiral modes.

Here we study the electron transport in samples where hDWs of different length $L$ are formed by electrostatic gating. Experimentally, in the limit $L \rightarrow 0$ only $11 \%$ of the edge current is diverted into the $\mathrm{hDW}$, a number drastically different from the $50 \%$ prediction for two noninteracting counter-propagating chiral channels. To address this discrepancy theoretically, we consider tunneling between Luttinger liquid modes ${ }^{30}$ through a $\mathrm{hDW}$ in the strong coupling limit ${ }^{31-33}$ and confirm that results remain the same if $\mathrm{hDW}$ is modeled as an extended object. We found that in the presence of a strong inter-edge tunneling edge channels in $u$ and $p$ regions populate unequally, both at the boundary of the $2 \mathrm{D}$ gas and within the hDW, forming a number of down- and upstream charge, spin, and neutral modes. For spin-conserving tunneling $1 / 4$ of the incoming charge current is diverted into the $\mathrm{hDW}$, while allowing spin-flip processes further reduces $\mathrm{hDW}$ current. Indeed, at high bias currents, we observe an increase in the current carried by the $\mathrm{hDW}$. This indicates the formation of a bottleneck for spin flips due to the Overhauser pumping of nuclei and a crossover from spin-non-conserving to spin-conserving transport.

\section{Experimental Results}

Several devices in a Hall bar geometry with multiple gates have been fabricated in order to study transport through hDWs, Fig. 1 (for heterostructure and fabrication details see Methods). Devices are separated into two regions $G 1$ and $G 2$; electron density $n$ in these regions can be controlled independently by electrostatic

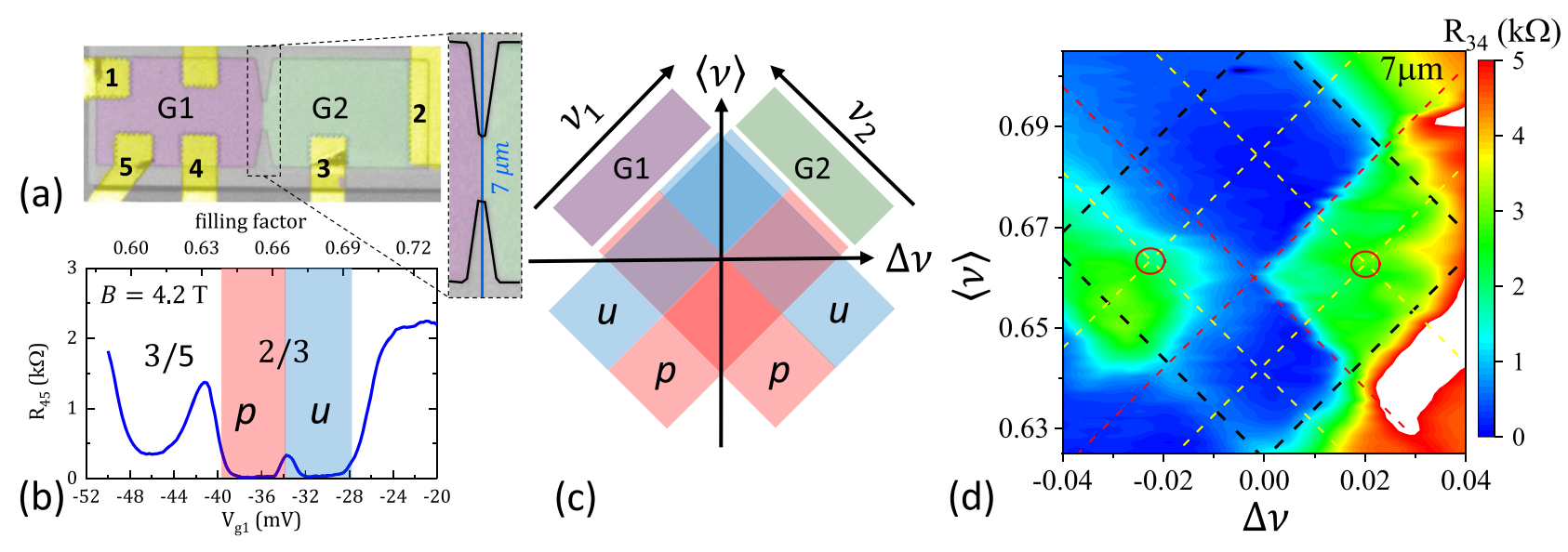

Fig. 1 Formation of helical domain walls at $\boldsymbol{\nu}=\mathbf{2}$ /3. a A false-color image of a typical device. Yellow regions are ohmic contacts, 2D gas in green and magenta regions is controlled by gates $\mathrm{G} 1$ and $\mathrm{G} 2$, in the gray area, 2D gas is removed. In the enlarged section, thick black lines outline the mesa boundary and a vertical blue line marks a physical boundary between $G 1$ and $G 2$. b Magnetoresistance $R_{45}=\left(V_{5}-V_{4}\right) / /$ of a $2 \mathrm{D}$ gas is plotted as a function of gate voltage (controlling filling factor $\nu$ ) at a fixed $B=4.2 \mathrm{~T}$. Small peak at $-34 \mathrm{mV}$ is the phase transition between unpolarized $(u)$ and polarized $(p) \nu=2 / 3$ FQHE liquids. c A diagram of $u$ and $p$ states as a function of $\nu_{1}$ and $\nu_{2}$ under gates $G 1$ and $G 2 ;\langle\nu\rangle=\left(\nu_{1}+\nu_{2}\right) / 2$ and $\Delta \nu=\left(\nu_{1}-\nu_{2}\right)$. d Resistance $R_{34}=\left(V_{4}-V_{3}\right) / /$ across a $7 \mu \mathrm{m}$-long gates boundary is plotted as a function of $\langle\nu\rangle$ and $\Delta \nu$. The black square outlines the $\nu=2 / 3$ region, red lines mark $u-p$ transitions and yellow lines mark centers of $u$ and $p$ regions. 

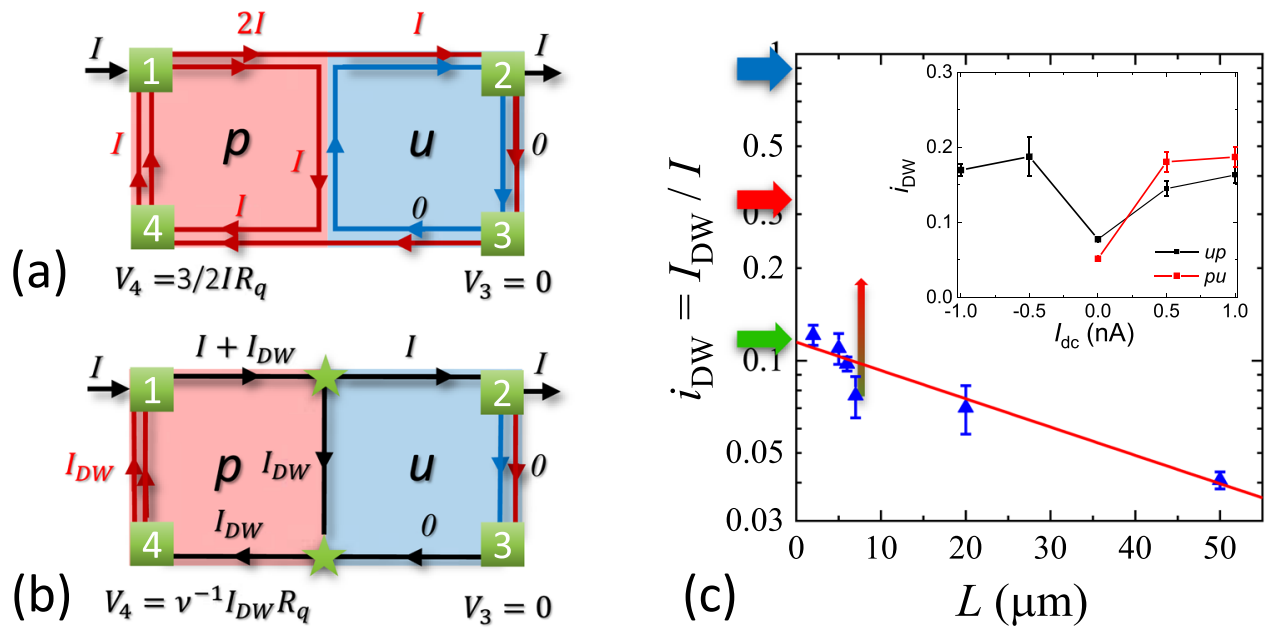

Fig. 2 Conduction of helical domain walls. a A simplified picture of noninteracting chiral edge modes at $\nu=2 / 3$. An inner spin-up edge (red) in $p$ state carries current $/$ while a spin-down edge (blue) in $u$ state carries no current. $\mathbf{b}$ Charge conservation and chirality of edge states set the potential $V_{4}=\nu^{-1} / \mathrm{DW} R_{q}$ to be proportional to the current $I_{\mathrm{DW}}$ diverted via the helical domain wall. c Scaling of the domain wall current $i_{\mathrm{DW}}=I_{\mathrm{DW}} / I$ with hDWs length $L$. The values are averaged between up and pu states (within red circles in Fig. 1d), error bars are standard deviations. Red line is a fit to an exponential decay with the $L=0$ value $i_{\mathrm{DW}}^{0}=0.115$ and the decay length $L_{0}=47 \mu \mathrm{m}$. Arrows indicate $i_{\mathrm{DW}}^{0}$ values expected for naive noninteracting edge model (blue) and Luttinger liquid model in the absence of spin-flip (red) and at spin-flip probability $r=3 / 4$ (green), see text for details. Vertical arrow marks $i_{\mathrm{DW}}$ shift when $I_{\mathrm{dc}}=1 \mathrm{nA}$ is applied. In the inset $i_{\text {DW }}$ dependence on large external dc current is plotted for $7 \mu \mathrm{m} \mathrm{hDW}$ for up and pu gates configuration right after the dc current is applied and before a measurable build-up of nuclear polarization.

gates. In the IQHE regime when filling factors $v$ under gates $G 1$ and G2 differ by one, a single chiral channel is formed along the gates boundary and resistance $R_{34}$ measured across the boundary is either quantized or zero depending on the sign of the filling factor gradient and direction of $B$ (see Supplementary Note 2). Likewise, a chiral channel is formed when the gates boundary separates two different FQHE states.

Fractional QHE states can be understood as integer QHE states for composite fermions in a reduced field, $v=v^{*} /\left(2 v^{*} \pm 1\right)$, where $v^{*}$ is the filling factor for composite fermions ${ }^{34}$. The energy separation between composite fermions levels depends on the competition between charging energy $E_{c} \propto \sqrt{B}$ and Zeeman energy $E_{Z} \propto B$, and the two lowest energy levels with opposite spins 0 -down and 1-up cross at finite $B^{*}>0$ due to different field dependencies. When the level crossing occurs within the $v=2 / 3$ plateau $\left(v^{*}=2\right.$ for composite fermions), the energy gap for quasiparticle excitations vanishes providing a mechanism for charge backscattering and, hence, at $B=B^{*}$ resistance of the $2 \mathrm{D}$ gas is no longer zero. In our devices, it is possible to control $B^{*}$ by electrostatic gating, and a small peak within the $2 / 3$ plateau in Fig. $1 \mathrm{~b}$ is a quantum Hall ferromagnetic transition between polarized $(p)$ and unpolarized $(u)$ regions.

Independent control of filling factors $v_{1}$ and $v_{2}$ under G1 and G2 divides the $2 / 3$ region into four quadrants $u u, p p$, $u p$, and $p u$, where the first letter corresponds to a polarization of the state under G1 and the second corresponds to polarization under G2. Within the Landauer-Büttiker formalism ${ }^{35,36}$ resistance $R_{34}=$ $\left(1 / v_{1}-1 / v_{2}\right) R_{q}$ should be zero for all combinations of polarizations under the gates since quantum numbers $v_{1}=v_{2}=2 / 3$ for both $u$ and $p$ states (here $R_{q}=h / e^{2}, h$ is the Plank's constant and $e$ is an electron charge). Experimentally $R_{34}$ is found to be vanishingly small in $u u$ and $p p$ quadrants, consistent with a single topological state being extended over the whole device. $R_{34}>0$ in $u p$ and $p u$ quadrants indicates backscattering between edge channels and, combined with zero longitudinal resistance under G1 and G2, reflects the formation of a conducting channel along the gates boundary. Unlike resistance measured across chiral channels formed, e.g., between $v=2 / 3$ and $v=3 / 5$ FQHE states (see Supplementary Note 2), the resistance measured across the boundary of $u$ and $p$ quantum liquids at $v=2 / 3$ shows almost no dependence on the direction of the external magnetic field and density gradient, consistent with the formation of a $\mathrm{hDW}^{37}$.

Protection of helical states from backscattering and localization is weaker for spatially separated chiral edge states, and conduction of hDWs is length-dependent. In Fig. 2a fraction of the external current $I$ that flows through the $\mathrm{hDW}, i_{\mathrm{DW}}=I_{\mathrm{DW}} / I$, is plotted as a function of hDW length $L . i_{\mathrm{DW}}$ is found to decrease exponentially with $L, i_{\mathrm{DW}}=i_{\mathrm{DW}}^{0} \exp \left[-L / L_{0}\right]$, with a characteristic length $L_{0}=47 \mu \mathrm{m}$. The value of $i_{\mathrm{DW}}^{0}$ corresponds to the transport through a ballistic $\mathrm{hDW}$ in the absence of localization. Within a simplified model of $v=2 / 3$ edge states consisting of equally populated $1 / 3+1 / 3$ chiral modes and no interaction between chiral channels with opposite spin polarization (Fig. 2a), one expects $i_{\mathrm{DW}}^{0}=1$ (marked by a blue arrow in Fig. 2c), an order of magnitude larger than the experimentally observed value (marked by a green arrow). Note that transport through helical modes formed in double quantum well structures are well described by this simple model of weakly interacting chiral states ${ }^{10}$.

\section{Theory}

An isolated hDW at a boundary of $p$ and $u$ phases was studied in refs. 19,37, where disk and torus geometries were employed to avoid physical edges of the sample and coupling of domain wall modes to these edges. Analytical model and numerical results indicate the existence of modes with opposite velocities and spins within the hDW region, a prerequisite for generating topological superconductivity. No neutral or spin modes appear within the $\mathrm{K}$-matrix Luttinger liquid approach ${ }^{30}$ in these isolated $\mathrm{hDW}$ models.

To calculate the scattering of edge modes at a sample boundary by a $\mathrm{hDW}$ we need to move beyond an isolated $\mathrm{hDW}$ model. A conventional starting point for chiral edge states description are two filled $\Lambda$-levels of composite fermions with equal or opposite spin in the $p$ and $u$ phases, with edge modes described by densities $\Phi_{p 1 \uparrow}, \Phi_{p 2 \uparrow}$ and $\Phi_{u 1 \uparrow}, \Phi_{u 2 \downarrow}$ correspondingly. As shown in Supplementary Note 3 , applying unitary transformations, we arrive to the description in terms of the separated charged and neutral modes 
$\varphi_{p c}=\left(\Phi_{p 1 \uparrow}+\Phi_{p 2 \uparrow}\right) / 3 \sqrt{2}$ and $\varphi_{p n}=\left(\Phi_{p 1 \uparrow}-\Phi_{p 2 \uparrow}\right) / \sqrt{2}$ for the $p$ phase, and separated charged and spin modes $\varphi_{u c}=\left(\Phi_{u 1 \uparrow}+\right.$ $\left.\Phi_{u 2 \downarrow}\right) / 3 \sqrt{2}$ and $\varphi_{u s}=\left(\Phi_{u 1 \uparrow}-\Phi_{u 2 \downarrow}\right) / \sqrt{2}$ for the $u$ phase, equivalent to the composite fermion description. The Luttinger liquid action for $v=2 / 3$ edge states, e.g., for the $u$ phase in terms of separated charge and spin modes, reads

$$
S=\frac{1}{4 \pi} \int d t \int d x\left[-3 \partial_{x} \varphi_{u c}\left(\partial_{t}+v_{c} \partial_{x}\right) \varphi_{u c}+\partial_{x} \varphi_{u s}\left(\partial_{t}-v_{s} \partial_{x}\right) \varphi_{u s}\right],
$$

where $v_{\mathrm{c}}$ and $v_{\mathrm{s}}$ are velocities of charge and spin modes, correspondingly. The spin mode determines the spin current in $u$ phase. The $p$ phase is described by a similar action, in which the neutral $\varphi_{p n}$ mode that determines a difference in the occupation of the first two composite fermion $\Lambda$-levels enters instead of $\varphi_{u s}$, the velocity of neutral mode $v_{\mathrm{n}}$ enters instead of $v_{s}$, and $\varphi_{p c}$ appears instead of $\varphi_{u c}$. These actions coincide with the Kane, Fisher, and Polchinsky ${ }^{38}$ Luttinger liquid action expressed in terms of the charge and neutral fields, see also refs. ${ }^{30,39}$, in the absence of tunneling between composite fermion modes due to impurity scattering. Indeed, at the edges of the $u$ phase such tunneling is forbidden in the absence of spin-flip processes, and $u$ phase exhibits spin-charge separation with pure spin and charge modes moving in opposite directions ${ }^{40}$ as in Eq. (1). To treat both phases, which exhibit quantization of Hall resistance $3 / 2 h / e^{2}$ and similar longitudinal resistance characteristics, on equal footing, we assume that no scattering between different modes occurs along edges in the $p$ phase also. At the same time, we show in Supplementary Note 3 that scattering between quasiparticle modes inside the domain wall does not alter measurable currents. In both phases quantization of the Hall resistance and charge and neutral (spin) mode separation is a consequence of the long-range Coulomb interaction ${ }^{30}$.

We generalize the known solution for tunneling ${ }^{41}$ of the fractional QHE modes through the point contact to consideration of tunneling through finite length domain wall between $p$ and $u$ phases. Point contact tunneling carried by electrons with the same spin can be described by the tunnel Hamiltonian

$$
\mathcal{H}_{T}=-\tilde{t} \cos \left(\Phi_{p 1 \uparrow}-\Phi_{u \uparrow \uparrow}\right)=-\tilde{t} \cos \left(\frac{1}{\sqrt{2}}\left[3 \varphi_{p c}-\varphi_{p n}-3 \varphi_{u c}+\varphi_{u s}\right]\right) .
$$

Mapping of an edge-hDW-edge structure onto one-dimensional bosonic modes $\varphi(x)$ is shown schematically in Fig. 3a. In general, $\mathrm{hDW}$ and each region outside of the $\mathrm{hDW}$ may contain up to eight bosonic modes $\varphi_{\alpha \beta}^{\rightleftarrows}(x)$, where $\alpha=\{p, u\}, \beta=\{c, n / s\}$ and the superscript $\{\leftarrow, \rightarrow\}$ indicates the projection of the velocity $v_{\beta}$ on the $x$-axis. However, the chirality of edge channels reduces the number of bosonic modes to four. It is convenient to consider two outgoing charge modes $\varphi_{p c}^{\overrightarrow{ }}\left(x_{2}\right)$ and $\varphi_{u c}^{\leftarrow}\left(x_{1}\right)$ and two outgoing spin/ neutral modes $\varphi_{p n}^{\leftarrow}\left(x_{1}\right)$ and $\varphi_{u s}\left(x_{2}\right)$.

In the strong coupling limit $\tilde{t} \rightarrow \infty$, charge, neutral, and spin currents can be found by imposing the following boundary conditions on bosonic fields right outside of the hDW $\left[x_{1}, x_{2}\right]$ :

$$
\left(\begin{array}{c}
\varphi_{p c}\left(x_{2}+0\right) \\
\varphi_{u s}\left(x_{2}+0\right) \\
\varphi_{u c}^{\leftarrow}\left(x_{1}-0\right) \\
\varphi_{p n}^{\leftarrow}\left(x_{1}-0\right)
\end{array}\right)=\frac{1}{4}\left(\begin{array}{cccc}
1 & -1 & 3 & 1 \\
-3 & 3 & 3 & 1 \\
3 & 1 & 1 & -1 \\
3 & 1 & -3 & 3
\end{array}\right)\left(\begin{array}{l}
\varphi_{p c}\left(x_{1}-0\right) \\
\varphi_{u s}\left(x_{1}-0\right) \\
\varphi_{u c}^{\leftarrow}\left(x_{2}+0\right) \\
\varphi_{p n}^{\leftarrow}\left(x_{2}+0\right)
\end{array}\right),
$$

which connect all incoming modes at the right side and outgoing modes at the left side of the equation. Our principal result is that imposing strong coupling boundary conditions in a general case of a domain wall of finite length results in the same currents flowing outside the domain wall as for the models of singlejunction connecting edges on opposite sample boundaries, two junctions on opposite edges, and two junctions with scattering between same spin modes in between. Inside the domain wall, the chiral evolution of modes is controlled by the average voltage shifts at their corresponding boundaries. In the presence of voltage $V$, the only incoming mode changing due to charge injection in the $p$ phase is $\varphi_{p c}\left(x_{1}\right)$, characterized by an average induced current $\bar{j}=\frac{e^{2} V}{3 \pi \hbar}$.

When spin-flip processes are absent, it is convenient to discuss the results in terms of currents carried by $\Phi_{p 1}\left(\Phi_{u 1}\right)$ and quasiparticle $\chi_{p 2}=\left(\varphi_{p c}-\varphi_{p n}\right) / \sqrt{2}\left(\chi_{u 2}=\left(\varphi_{p c}-\varphi_{p s}\right) / \sqrt{2}\right)$ modes. We show that $\Phi_{p 1 \uparrow}\left(\Phi_{u 1 \uparrow}\right)$ modes propagate along the edges of the 2D gas and do not enter the domain wall, while $\chi_{p 2}$ and $\chi_{u 2}$ modes flow along the boundaries of $p$ and $u$ phases correspondingly, including inside the domain wall, as shown schematically in Fig. 3b. Notable features of our solution are unequal distribution of carried currents between the modes caused by strong coupling to the domain wall and the presence of spin current along the edge of the $u$ phase for $x>x_{2}$. The total current flowing along the $\mathrm{hDW} I_{\mathrm{DW}}=1 / 4\left(I+I_{\mathrm{DW}}\right)$ or $i_{\mathrm{DW}}=1 / 3$. This value is three times
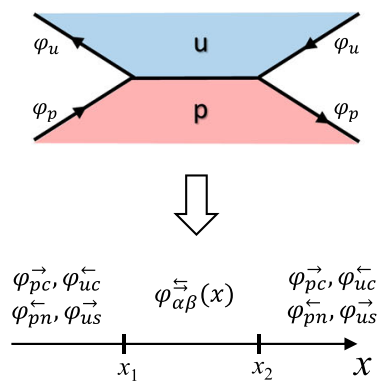

(a) (b)
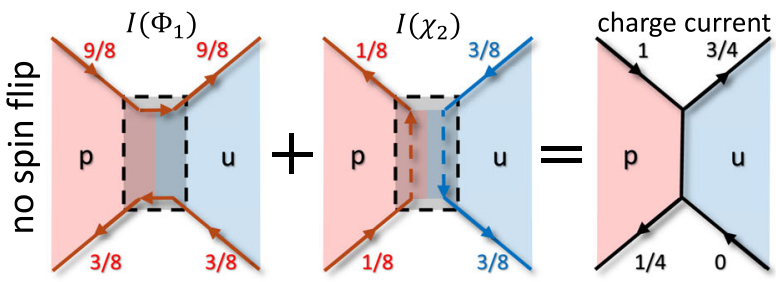

(c)

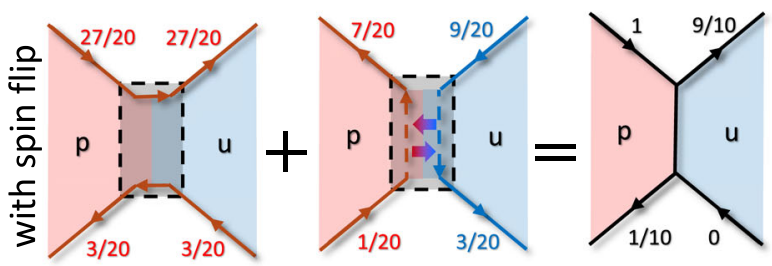

Fig. 3 Schematic representation of currents. a Mapping of bosonic modes $\varphi_{\alpha}$ along sample edges onto a 1D Luttinger model modes $\varphi_{\alpha \beta}^{\rightleftarrows}(x)$ for a domain wall with length $L=x_{2}-x_{1}$. Subscripts $\alpha=\{p, u\}$ label polarized and unpolarized phases, $\beta=\{c, n, s\}$ is for charge, neutral, and spin modes and an arrow in the superscript specifies projection of the mode's group velocity. Arrows on the edges define the chirality of edge channels. $\mathbf{b}$, $\mathbf{c} V i$ isualization of currents due to the propagation of $\Phi_{1}$ and $\chi_{2}$ modes without spin flips (b) and in the presence of spin flips with the probability $r=3 / 4$ (c). Red (blue) mode color indicates a spin-up (spin-down) polarization. Numbers indicate the fraction of the incoming current carried by the mode. Arrows correspond to directions of currents carried by corresponding modes. 
larger than the experimentally measured $i_{\mathrm{DW}}$ and is indicated by a red arrow in Fig. 2c.

In $2 \mathrm{D}$ gases formed in GaAs heterostructures spin transition at $v=2 / 3$ is accompanied by a dynamic nuclear spin polarization ${ }^{42}$. Its main mechanism is the hyperfine coupling of electron and nuclear spins, which for QHE plateaus is usually suppressed due to a large difference between electron and nuclear Zeeman splitting. Near the $u-p$ phase transition, however, electronic states with spin-up and spin-down are almost degenerate, enabling the hyperfine coupling. This spin-flip mechanism can lead to the scattering between $\chi_{p 2}$ and $\chi_{u 2}$ modes propagating inside the domain wall, see Supplementary Note 3 . Notably, $\Phi_{1}$ modes still propagate along the $2 \mathrm{D}$ gas boundary and do not enter the domain wall. However, conservation of total current carried by $\Phi_{1}$ and $\chi_{2}$ modes results in the current redistribution between the modes. The ratio characterizing the domain wall current $i_{\mathrm{DW}}(r)$ becomes a function of the spin-flip probability $r$ and changes continuously between $1 / 3$ for $r=0$ and zero for $r=1$. Experimentally measured values correspond to $r \approx 3 / 4$, corresponding currents are shown schematically in Fig. $3 \mathrm{c}$.

To test the role of spin flips it is possible to pass a large dc current and polarize nuclei in the vicinity of the tri-junction. Saturation of nuclear spin polarization is expected to create a bottleneck for electron spin flips and disable charge transfer between two $\chi_{2}$ bosonic modes with opposite polarization. Indeed, application of $I_{\mathrm{dc}}>0.5 \mathrm{nA}$ results in approximately a threefold increase of $i_{\mathrm{DW}}$, as shown in the inset in Fig. 2. A corresponding shift of $i_{\mathrm{DW}}$ for the $7 \mu \mathrm{m} \mathrm{hDW}$ is shown with a vertical arrow on the main plot. This shift is consistent with the triple current increase expected for the crossover from a spin-flipdominated to a no-spin-flip transport.

\section{Methods}

Devices are fabricated from GaAs/AlGaAs inverted single heterojunction heterostructures with electron gas density $0.9 \cdot 10^{11} \mathrm{~cm}^{-2}$ and mobility $5 \cdot 10^{6} \mathrm{~cm}^{2} / V s$. Details of heterostructure design can be found in ref. ${ }^{43}$, these heterostructures demonstrate efficient electrostatic control of the spin transition at $v=2 / 3$, see ref. ${ }^{37}$. Devices are patterned in a Hall bar geometry using e-beam lithography and wet etching, the photograph of a typical sample is shown in Fig. 1. Devices are divided into two regions by semitransparent gates $(10 \mathrm{~nm}$ of $\mathrm{Ti})$, the gates are separated from the surface of the wafer and from each other by $50 \mathrm{~nm}$ of $\mathrm{Al}_{2} \mathrm{O}_{3}$ grown by the atomic layer deposition (ALD). Gates boundary is aligned with a $2-50-\mu \mathrm{m}$-wide mesa constriction. Ohmic contacts are formed by annealing $\mathrm{Ni} / \mathrm{Ge} /$ Au $30 / 50 / 100 \mathrm{~nm}$ at $450{ }^{\circ} \mathrm{C}$ for $450 \mathrm{~s}$ in $\mathrm{H}_{2} / \mathrm{N}_{2}$ atmosphere. Measurements are performed in a dilution refrigerator at a base temperature of $20 \mathrm{mK}$ using conventional low-frequency lock-in technique with excitation current $I_{\mathrm{ac}}=100 \mathrm{pA}$. A $2 \mathrm{D}$ electron gas is formed by shining a red LED at $4 \mathrm{~K}$. More details on the $2 \mathrm{D}$ gas preparation and sample characterization can be found in Supplementary Note 1.

\section{Data availability}

The data that support the findings of this study are available from the corresponding author upon reasonable request.

Received: 8 January 2021; Accepted: 20 August 2021;

Published online: 07 September 2021

\section{References}

1. Lindner, N. H., Berg, E., Refael, G. \& Stern, A. Fractionalizing Majorana fermions: non-abelian statistics on the edges of abelian quantum hall states. Phys. Rev. X 2, 041002 (2012).

2. Clarke, D. J., Alicea, J. \& Shtengel, K. Exotic non-Abelian anyons from conventional fractional quantum Hall states. Nat. Commun. 4, 1348 (2012)

3. Alicea, J. \& Fendley, P. Topological phases with parafermions: theory and blueprints. Annu. Rev. Conden. Matter Phys. 7, 119 (2016).

4. Sarma S. D. \& Pinczuk, A. (eds) Perspectives in Quantum Hall Effects: Novel Quantum Liquids in Low-Dimensional Semiconductor Structures (Wiley-VCH Verlag GmbH, 2007)
5. Ando, T. \& Suzuura, H. Presence of perfectly conducting channel in metallic carbon nanotubes. J. Phys. Soc. Japan 71, 2753 (2002).

6. Xu, C. \& Moore, J. E. Stability of the quantum spin Hall effect: effects of interactions, disorder, and Z2 topology. Phys. Rev. B 73, 045322 (2006).

7. Bardarson, J. H. A proof of the Kramers degeneracy of transmission eigenvalues from antisymmetry of the scattering matrix. J. Phys. A Math. Theor. 41, 405203 (2008).

8. Young, A. F. et al. Tunable symmetry breaking and helical edge transport in a graphene quantum spin Hall state. Nature 505, 528 (2014).

9. Sanchez-Yamagishi, J. D. et al. Helical edge states and fractional quantum Hall effect in a graphene electron-hole bilayer. Nat. Nanotechnol. 12, 118 (2017).

10. Ronen, Y. et al. Robust integer and fractional helical modes in the quantum Hall effect. Nat. Phys. 14, 411 (2018).

11. Kazakov, A. et al. Electrostatic control of quantum Hall ferromagnetic transition: a step toward reconfigurable network of helical channels. Phys. Rev. B 94, 075309 (2016).

12. Kazakov, A. et al. Mesoscopic transport in electrostatically defined spin-full channels in quantum Hall ferromagnets. Phys. Rev. Lett. 119, 046803 (2017)

13. Simion, G. et al. Impurity-generated non-Abelions. Phys. Rev. B 97, 245107 (2018).

14. Jain, J. K. Composite fermion approach for the fractional quantum Hall effect. Phys. Rev. Lett. 63, 199 (1989).

15. Kukushkin, I. V., Klitzing, K. V. \& Eberl, K. Spin polarization of composite fermions: measurements of the fermi energy. Phys. Rev. Lett. 82, 3665 (1999).

16. Vaezi, A. Fractional topological superconductor with fractionalized Majorana fermions. Phys. Rev. B 87, 035132 (2013).

17. Mong, R. S. K. et al. Universal topological quantum computation from a superconductor-Abelian quantum hall heterostructure. Phys. Rev. X 4, 011036 (2014).

18. Vaezi, A. Superconducting analogue of the parafermion fractional quantum Hall states. Phys. Rev. X 4, 031009 (2014).

19. Liang, J., Simion, G. \& Lyanda-Geller, Y. Parafermions, induced edge states, and domain walls in fractional quantum Hall effect spin transitions. Phys. Rev. B 100, 075155 (2019).

20. Gurman, I. et al. Extracting net current from an upstream neutral mode in the fractional quantum Hall regime. Nat. Commun. 3, 1289 (2012).

21. Inoue, $\mathrm{H}$. et al. Proliferation of neutral modes in fractional quantum Hall states. Nat. Commun. 5, 4067 (2014).

22. Rosenblatt, A. et al. Transmission of heat modes across a potential barrier. Nat. Commun. 8, 2251 (2017)

23. Wang, J., Meir, Y. \& Gefen, Y. Edge reconstruction in the $v=2 / 3$ fractional quantum Hall state. Phys. Rev. Lett. 111, 246803 (2013).

24. Lafont, F., Rosenblatt, A., Heiblum, M. \& Umansky, V. Counter-propagating charge transport in the quantum Hall effect regime. Science 363, 54 (2019).

25. Bid, A. et al. Shot noise and charge at the $v=2 / 3$ composite fractional quantum Hall state. Phys. Rev. Lett. 103, 236802 (2009).

26. Kronmüller, $\mathrm{S}$. et al. New resistance maxima in the fractional quantum Hall effect regime. Phys. Rev. Lett. 81, 2526 (1998).

27. Kraus, S. et al. From quantum Hall ferromagnetism to huge longitudinal resistance at the $v=2 / 3$ fractional quantum Hall state. Phys. Rev. Lett. 89, 266801 (2002)

28. Stern, O. et al. NMR study of the electron spin polarization in the fractional quantum Hall effect of a single quantum well: spectroscopic evidence for domain formation. Phys. Rev. B 70, 075318 (2004).

29. Cohen, Y. et al. Synthesizing a $v=2 / 3$ fractional quantum Hall effect edge state from counter-propagating $v=1$ and $v=1 / 3$ states. Nat. Commun. 10, 1920 (2019).

30. Wen, X.-G. Topological orders and edge excitations in fractional quantum Hall states. Adv. Phys. 44, 405 (1995).

31. Sandler, N. P., de C. Chamon, C. \& Fradkin, E. Noise measurements and fractional charge in fractional quantum Hall liquids. Phys. Rev. B 59, 12521 (1999).

32. Nayak, C., Fisher, M. P. A., Ludwig, A. W. W. \& Lin, H. H. Resonant multilead point-contact tunneling. Phys. Rev. B 59, 15694 (1999).

33. Moore, J. E. \& Wen, X.-G. Critical points in edge tunneling between generic fractional quantum Hall states. Phys. Rev. B 66, 115305 (2002).

34. Jain, J. K. Composite Fermions (Cambridge Univ. Press, 2007).

35. Beenakker, C. W. J. Edge channels for the fractional quantum Hall effect. Phys. Rev. Lett. 64, 216 (1990).

36. Brey, L. Edge states of composite fermions. Phys. Rev. B 50, 11861 (1994)

37. Wu, T. et al. Formation of helical domain walls in the fractional quantum Hall regime as a step toward realization of high-order non-Abelian excitations. Phys. Rev. B 97, 245304 (2018).

38. Kane, C. L., Fisher, M. P. A. \& Polchinski, J. Randomness at the edge: theory of quantum Hall transport at filling $v=2 / 3$. Phys. Rev. Lett. 72, 4129 (1994).

39. Kane, C. L \& Fisher, M. P. A. Perspectives in Quantum Hall Effects (WileyVerlag GmbH, 1997). 
40. Wu, Y.-H., Sreejith, G. J. \& Jain, J. K. Microscopic study of edge excitations of spin-polarized and spin-unpolarized $v=2 / 3$ fractional quantum Hall effect. Phys. Rev. B 86, 115127 (2012).

41. Fendley, P., Ludwig, A. W. W. \& Saleur, H. Exact nonequilibrium dc shot noise in Luttinger liquids and fractional quantum Hall devices. Phys. Rev. Lett. 75, 2196 (1995).

42. Li, Y. Q. \& Smet, J. H. in Spin Physics in Semiconductors. Ch 12, 1st. edn. (ed. Dyakonov, M. I.) (Springer, 2008).

43. Wan, Z. et al. Induced superconductivity in high-mobility two-dimensional electron gas in gallium arsenide heterostructures. Nat. Commun. 6, 7426 (2015).

\section{Acknowledgements}

The experimental part of the work is supported by NSF award DMR-1836758 (Y.W. and L.P.R.). Theoretical work is supported by the US Department of Energy, Office of Basic Energy Sciences, Division of Materials Sciences and Engineering under Award DESC0010544 (Y.L.-G.). Heterostructures development and growth is funded in part by the Gordon and Betty Moore Foundation's EPiQS Initiative, Grant GBMF9615 to L.N.P., and by the NSF MRSEC grant DMR-1420541.

\section{Author contributions}

L.P.R. conceived, Y.W. and Z.W. performed experiments, V.P. and Y.L.-G. developed theory, K.W.W., K.W.B., and L.N.P. developed and grew heterostructure materials. Y.W., V.P., Y.L.-G., and L.P.R. have written the manuscript.

\section{Competing interests}

The authors declare no competing interests.

\section{Additional information}

Supplementary information The online version contains supplementary material available at https://doi.org/10.1038/s41467-021-25631-2.

Correspondence and requests for materials should be addressed to Y.L-G. or L.P.R.

Peer review information Nature Communications thanks the anonymous reviewers for their contribution to the peer review of this work. Peer reviewer reports are available.

Reprints and permission information is available at http://www.nature.com/reprints

Publisher's note Springer Nature remains neutral with regard to jurisdictional claims in published maps and institutional affiliations.

\begin{abstract}
cC) (i) Open Access This article is licensed under a Creative Commons Attribution 4.0 International License, which permits use, sharing, adaptation, distribution and reproduction in any medium or format, as long as you give appropriate credit to the original author(s) and the source, provide a link to the Creative Commons license, and indicate if changes were made. The images or other third party material in this article are included in the article's Creative Commons license, unless indicated otherwise in a credit line to the material. If material is not included in the article's Creative Commons license and your intended use is not permitted by statutory regulation or exceeds the permitted use, you will need to obtain permission directly from the copyright holder. To view a copy of this license, visit http://creativecommons.org/ licenses/by/4.0/.
\end{abstract}

(C) The Author(s) 2021 